\title{
RÉGION DU SUD-OUEST
}

\begin{tabular}{|c|c|c|c|c|c|c|c|}
\hline $\mathrm{N}^{\circ}$ & COURS D'EAU & NOMS DES USINES & PROPRIÉTAIRES & PUISSAN & $\begin{array}{l}\text { NORMAI } \\
\text { ES USINE }\end{array}$ & EN KW: & $\begin{array}{r}\text { REGIME } \\
\text { A-Autorisation. }\end{array}$ \\
\hline d'ordro & 2 & 3 & $\begin{array}{l}4 \\
4\end{array}$ & $\begin{array}{c}\text { en } \\
\text { service } \\
5\end{array}$ & $\begin{array}{l}\text { en cons- } \\
\text { truction } \\
\quad 6\end{array}$ & $\begin{array}{c}\text { en } \\
\text { projet } \\
7\end{array}$ & $\begin{array}{c}\mathrm{C}-\text { Connession et date d } \\
\text { réglementation. } \\
8\end{array}$ \\
\hline
\end{tabular}

BASSIN DE I'EFRAUIT

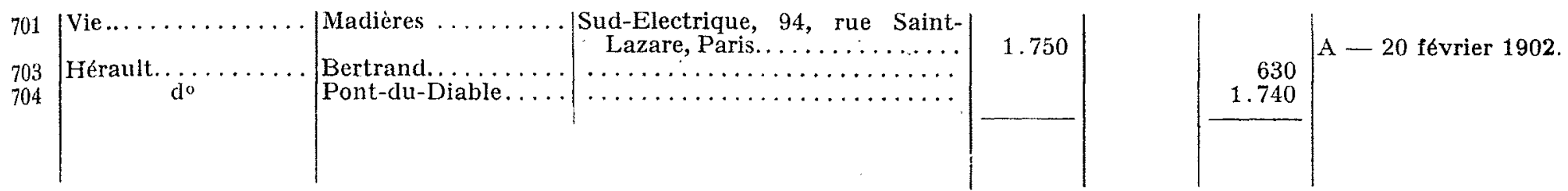

\section{BASSIN DE IOARB}

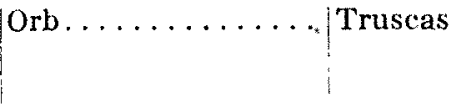

Société Biterroise de Force et Lumière, 12, r. St-Florentin, Paris $\frac{520}{520}$
Non réglementée

BASSIIV DE IUATIE

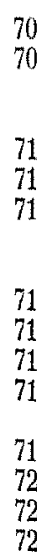

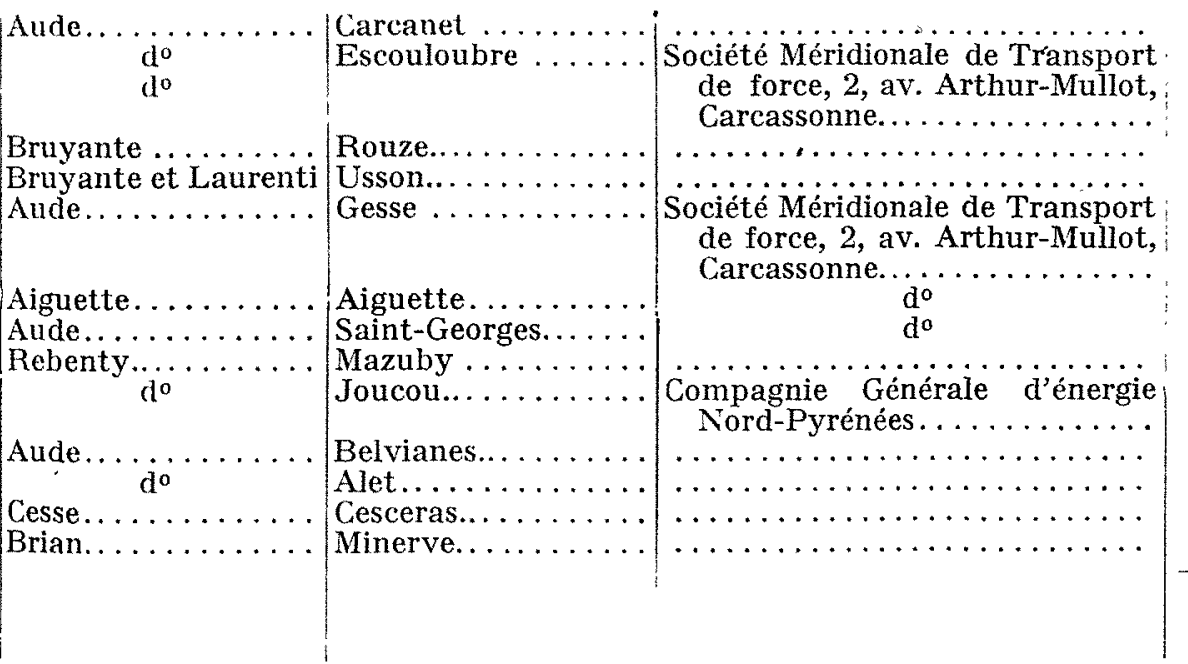

En instance de con1.400 2.780 cession.

510

580

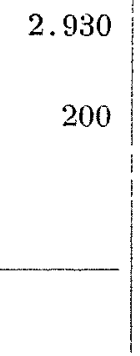

A - 23 juin 1906 .

A - +1 juillet 1899 .

A - 15 février 1917

\section{BASSIN DE IA TET}

728
730
731
732
738
734
735
736
738
739
7

731
732
733
734
735
736
737
738
739
7

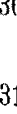

Tet. ........... Aveillane ........

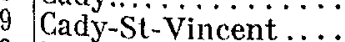

Tet.

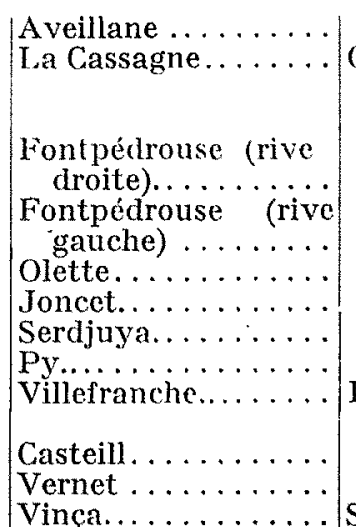

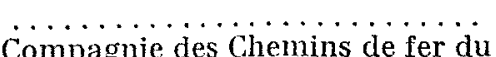
Midi, 54, boulev. Haussmann,

Paris ............... 2.150

do $^{\circ}$

1.730

$d^{\prime \prime}$

..................

$\ldots \ldots \ldots \ldots \ldots \ldots \ldots \ldots$

Industrie électrique Ecoiffier, 37 quai Vauban, Perpignan.......

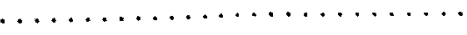

Sociète Hydro-électrique Roussil lonnaise, cité Partissol, Perpignan
3.270

L'arrêté d'autorisation n'a pas été pris 8 juin 1917.

12.840

2.220

1.400

850

870

C. 1 aoitt 1860

630 


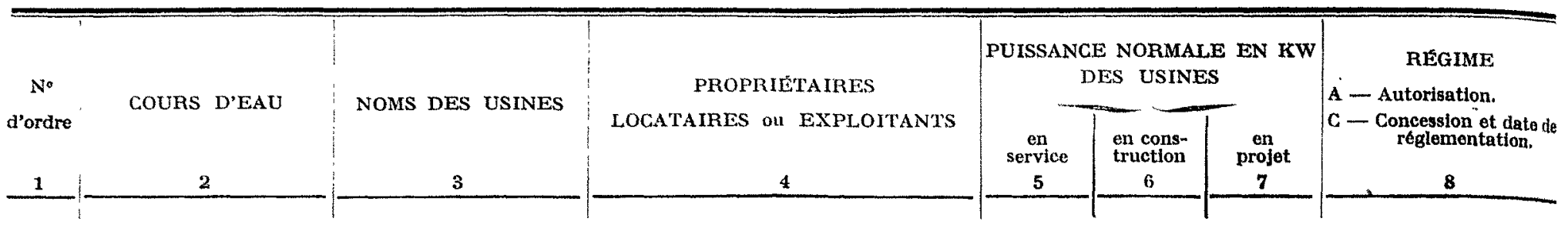

\section{BASSIN DU TECF}

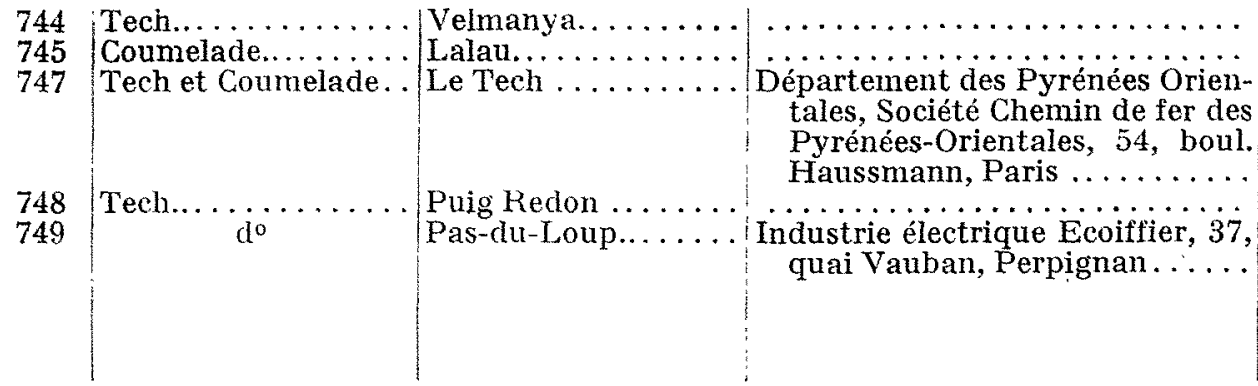

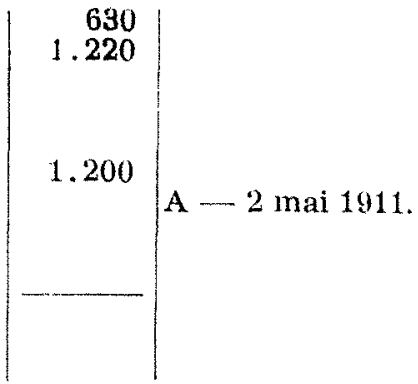

BASSIN DE IA GAROIVINE
751

753

754

755

757

758
759

763

764

765

766

Neste d'Aure......... Lavedan ............

Neste de Carabide... Caillaouas... . . . . . . .

773

774

776

780

781

783

784

785

786

790

792

794
795

796

797

798

800

801

Garonne.

Pique

$\mathrm{d}^{\mathrm{o}}$

Neste d'oc..........

Ons..............

$\mathrm{d}^{\mathrm{o}}$

este du Louron...

$d^{0}$

Louvendille... . . . . . . . . . . . . .

Neste ...........

do

$d^{\circ}$

Garonne.

do $^{\circ}$

do

do

do

$\mathrm{d}^{\circ}$

Salat et Cougnets

Alet...

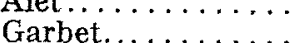

dio

Salat................

i.

Loz......

$\mathrm{d}^{0}$

do

Saint-Péat

Pique inf. . . . . . . .

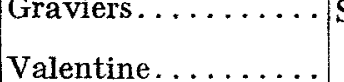

Facourt ............

La Moulasse......... do
Compagnie d'électricité industr 41, boulev. Haussmann, Paris. do

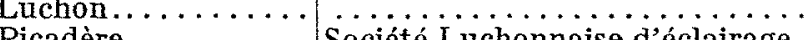

icadère.......... Société Luchonnaise d'éclairage.

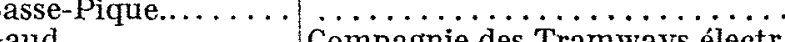
Marignac-Val-d'Aran, 38, ch.

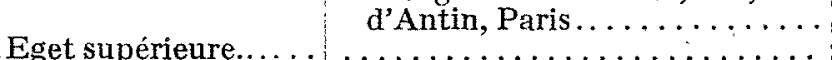

Eget inférieure..... Compagnie des Chemins de fer du Midi, 54, Boulevard Haussmann,

Paris ..................

Société Minière et Métallurgique de Penarroya, 12, place Ven-

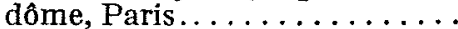

$\ldots \ldots \ldots \ldots \ldots \ldots \ldots$

$\ldots \ldots \ldots \ldots \ldots \ldots \ldots$

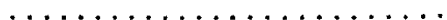

$d^{0}$ Ouest, 12, r. Roquépine, Paris.

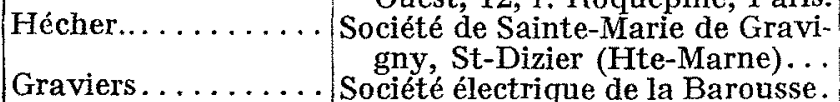

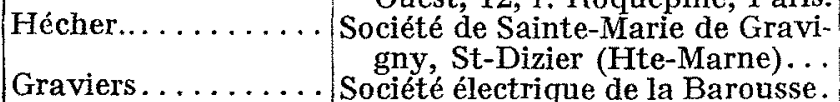

Viconte ......... M. Léon Lauvaux, à LabartheInard.

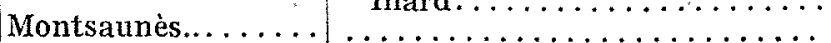

Apas......... $\quad$ Sirven, 76 , rue de la Colombette, Toulouse . . . . . . . . .

\begin{tabular}{|c|c|} 
Mandoux .......... Forces Motrices de la Garonne, \\
P2, boulev. Haussmann, Paris.
\end{tabular}

\begin{tabular}{|c|c|} 
Mandoux .......... Forces Motrices de la Garonne, \\
P2, boulev. Haussmann, Paris.
\end{tabular}

Salau............ Société des Papeteries de Lédar,

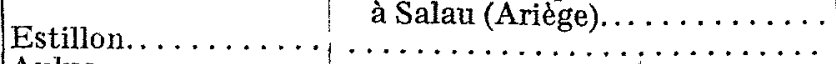

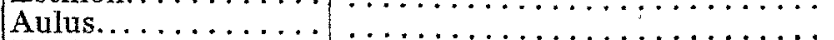

Ercé.......... Compagnie des Tramways de $\mathrm{l}$ Ariège à Saint-Girons. . . . . . . .

miege a Saint-Girons..........

Société Job, boulevard de Strasbourg, Toulouse............ Arrout ............ Compagnie des Tramways de do
8.380

En instance de réglementatio $n$.

5.860 $\mathrm{d}^{\circ}$

2.030

270

9.400

10.450

4.600

4.950

5.000

730

220

390

770

1.390

400

A p. -2 mai 1901.

A -19 janvier 1916

$\mathrm{C}-17$ juillet 1908.

A -- 15 avril 1918.

2.060

6.660

7.000

A -28 avril 1919.

A -28 avril 1919 .

C - 12 janvier 1918.

En cours de réglem.

C - 21 février 1863 ,

25.000

$\mathrm{A}-28$ décem.1905.

15.200

Existence légale.

C -26 février 1835 .

C -28 janv. 1905 .

6.600

A - 1 er mars 1902.

440
3.320

4.700

A -22 mai 1911

4.080

A -7 janvier 1899 .

$\mathrm{A}-2$ décemb.1876,

$A=14$ mai 1910

800 


\begin{tabular}{|c|c|c|c|c|c|c|c|}
\hline $\mathrm{N}^{0}$ & COUAS D'EAU & NOMS DES LSINES & PROPRIETAIRES & PUISSAN & $\begin{array}{l}\text { NORMA } \\
\text { ES USIN }\end{array}$ & EN KW & $\begin{array}{l}\text { REFIME } \\
\text { A - Autorisation. }\end{array}$ \\
\hline d'ordre & 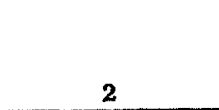 & 3 & $\begin{array}{l}\text { LOCATAIRES OU EXPLOITANTS } \\
\qquad 4\end{array}$ & $\begin{array}{c}\text { en } \\
\text { service } \\
\mathbf{5}\end{array}$ & $\begin{array}{l}\text { en cons- } \\
\text { truction } \\
\quad 6\end{array}$ & $\begin{array}{l}\text { en } \\
\text { projet } \\
7\end{array}$ & $\begin{array}{c}\text { C- Concession et date do } \\
\text { réglementation. } \\
8\end{array}$ \\
\hline
\end{tabular}

BASSIN DE LA GARONNE (suite)

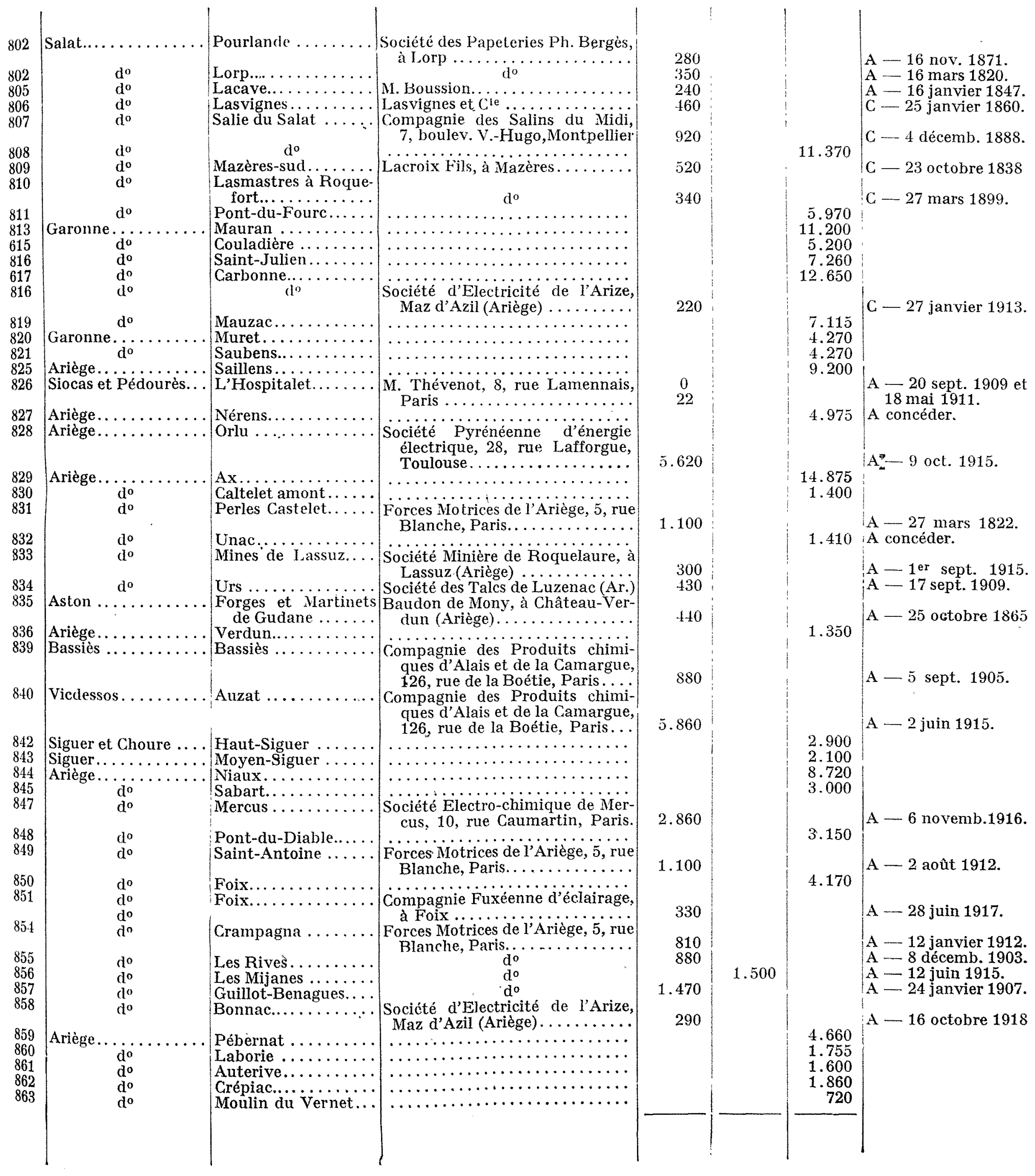




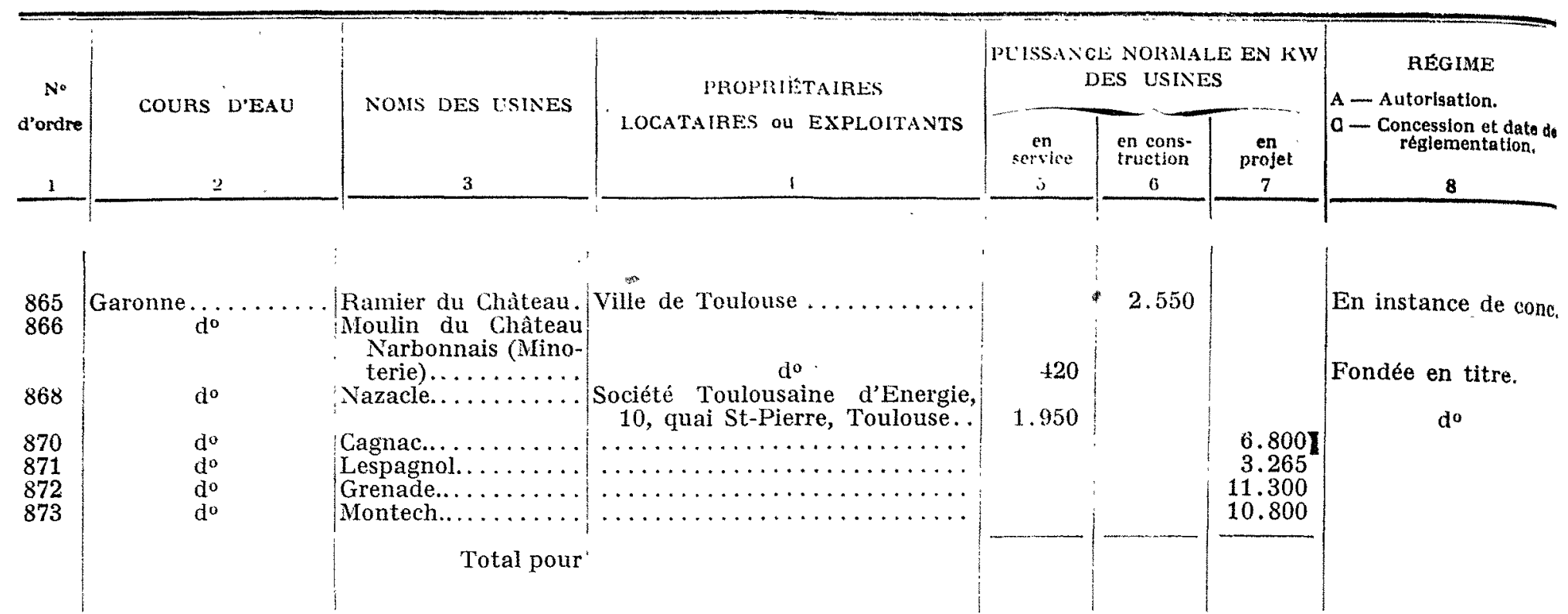

\section{BASSIN DU TARIV}

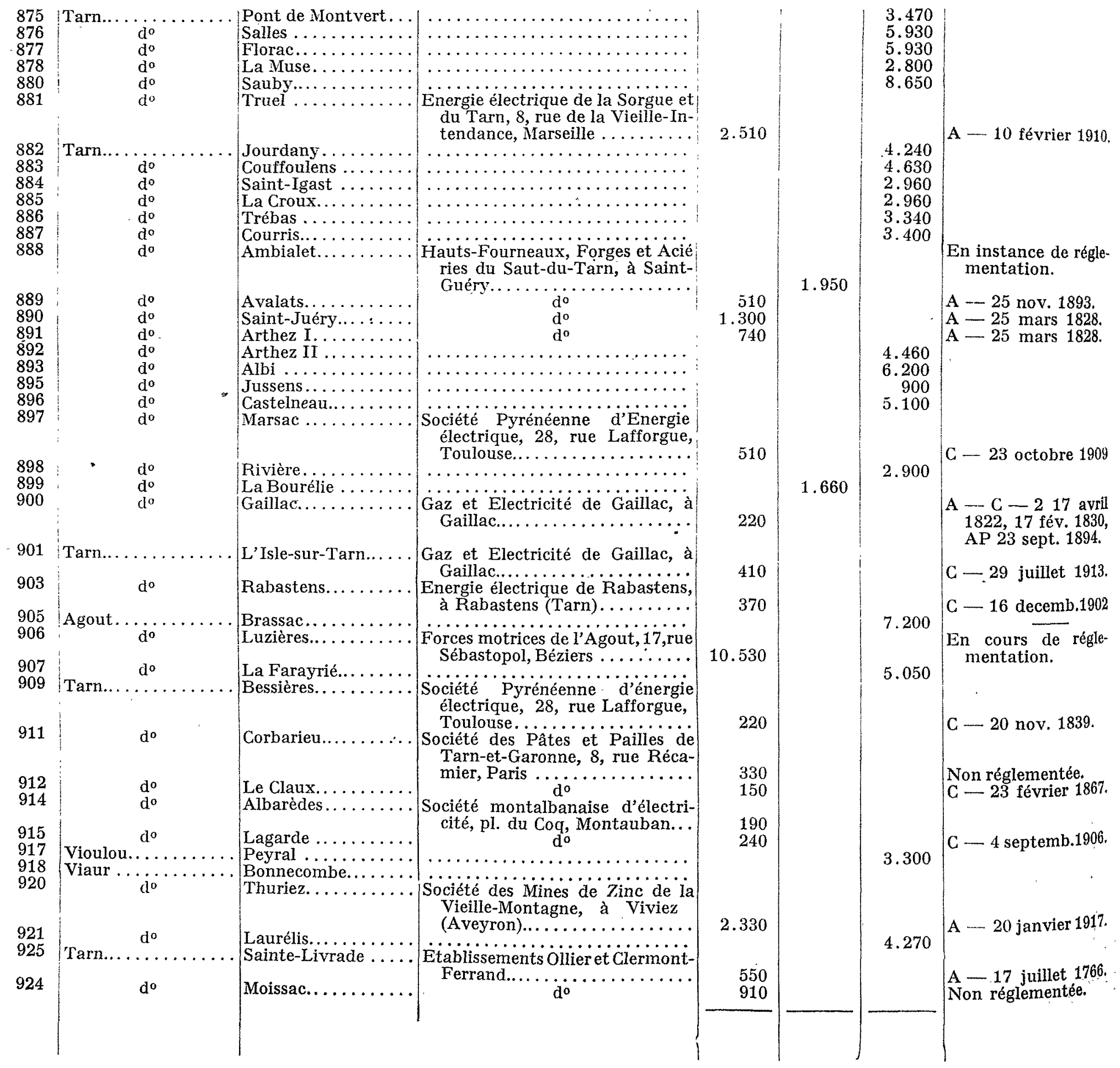

